\title{
A Novel Non-Invasive Algorithm Using the concept of Nail Based Anemia Disease Detection
}

\author{
D.O.I - 10.51201/Jusst12611 \\ http://doi.org/10.51201/Jusst12611
}

\author{
Saurabh Mitra, Dr. Shanti Rathore, Dr. Sanjeev Kumar Gupta \\ Research Scholar, Associate Professor, Dean \\ Dept. of Electronics \& Communication Engg \\ Dr. C. V. Raman University Bilaspur (C.G.), Rabindranath Tagore University Bhopal (M.P.)
}

\begin{abstract}
Anemia is a danger disease for the human life. If anemia diagnosis is not found in time than its very difficult to recover the patient specially in COVID-19 time it's a deadly disease. As we know in this era 2020 COVID is create a huge change in human life that's why after 2019 is called New life. As we know there is lots of approaches are there to identify the anemia, but there is very few approaches are there which are non-invasive, and those approaches are not good in terms of the quality of the result and most important they are not a good real time analysis system. So, in this paper we proposed a novel non-invasive algorithm which is able to detect the anemia using the human nails. In this approach we use computer vision, machine and deep learning concept and based on that only we decide the anemia level on any particular patient. Our propose approach is complete real time and this system is able to provide result in very less time. Key Words:Invasive, NonInvasive, SPO2, Hardware, Device.
\end{abstract}

\section{INTRODUCTION}

Tainting with extraordinary serious respiratory issue Covid 2 (SARS-COV-2) routinely achieves Coronavirus contamination 2019 (COVID-19), a disorder that endangers unreasonably the old, those with earlier tenacious conditions, for instance, cardiovascular disease, diabetes mellitus and hypertension $(1,2)$. In the occasion that separating, Covid-19 can incite sepsis, septic stagger, and different organ brokenness condition, with mechanical ventilation or extracorporeal film oxygenation having low accommodating efficacy (3). The pathophysiological establishment shrouded rot and low suitability of ordinary meds is obfuscated. Most patients with COVID-19 who require genuine thought will develop an atypical sort of the extraordinary difficulty respiratory confusion (ARDS) with protected lung gas volume (4), proposing hypoxia in view of physiological cycles other than alveolar brokenness may accept a capacity in the expectation of the disease (5). Upset iron absorption may be one such impacted measure. Undoubtedly, late data show that COVID-19 patients will when all is said in done present reduced hemoglobin levels exhibiting the presence of feebleness, and neurotically extended levels of ferritin. An examination of 67 COVID-19 patients in Singapore definite that during their course in a crisis unit), (patients developed more critical and basically lower hemoglobin levels, appeared differently in relation to patients not admitted to ICU (6). Another examination in more seasoned patients hospitalized for COVID-19 found that most patients had hemoglobin levels lower than the common reach, yet didn't find basic differences in hemoglobin levels among survivors and non-survivors. Regardless, advancement was divided for half of the patients (7). In a report of 5700 patients hospitalized for COVID-19 in the New York City locale, ferritin levels were neurotically high, a finding as per past examinations from China $(8,9)$. Both whiteness and hyperferritenemia, paying little brain to the shrouded pathology, are strong markers of mortality $(10,11)$. Paleness could be the outcome of iron-bound erythropoiesis arising out of changes in iron processing. Extended ferritin levels could be quality of a strong provocative reaction in COVID-19 or related to viral entry into the human body and its impact on iron metabolism $(12,13)$. Iron is a key micronutrient for the two individuals and pathogens $(14)$. The inalienable safe response could restrict iron availability during illnesses to deny its microorganism, an instrument that would moreover provoke sickliness $(15,16)$. Shortcoming, in this way, diminishes oxygen transport to the tissue and may likewise accept a huge part in the new development of multi organ dissatisfaction. Thusly, it is huge to understand the association between sickliness, iron absorption and development of COVID-19, and whether these affiliations shift by age, sex and presence of progressing conditions. A New Method and a Non-Invasive Device to Estimate Anemia Based on Digital Images of the Conjunctiva Anemia is a general clinical issue with tremendous ramifications for human flourishing. The World Health Organization (WHO) evaluates that around two billion individuals are iron lacking, which is dened as $\mathrm{Hb}$ focuses that are under the suggested edges [17], [18]. Weakness is dened as a lessening in the red platelets drifting in the blood or as an abatement in the centralization of $\mathrm{Hb}$ : subsequently, it is a reduced restriction of the blood to move oxygen. It begins in a general sense from solid components (iron deciency, enhancements and minerals), overpowering diseases (wild fever, intestinal parasites) or hereditary fragments (hemoglobin opathies) [19], [20]. These variables can happen in partition yet are continually related. Iron deciency Anemia is the most striking dietary deciency, and it causes innumerable passings (allianceanemia.org); it is in addition committed for expanded unpleasantness and mortality in pre-more young understudies and pregnant ladies. In 2002, iron deciency Anemia was viewed as among the principle contributing parts to the general heap of difficulty [21]. Deficiency 
has a moderate progress: ordinarily, no conspicuous signs show up for $\mathrm{Hb}>9-10 \mathrm{~g} / \mathrm{dl}$, since the human body executes compensatory parts, for example, developing the extent of blood directed, with the objective that the extent of oxygen delivered to the tissues remains essentially unaltered. Right when the remuneration can now don't ensure a decent portion of oxygen, signs, for example, deficiency, whiteness, crabbiness, expanded heartbeat, absence of rest, cerebral misery and different others show up. The clinical report, if all else fails, is questionable and difcult to depict. Similarly, Anemia is reliably not separated or enough treated in ceaseless patients for whom it is a basic hazard factor (checking mortality) and affects the individual satisfaction. In spite of its etiology, since an incredible level of Anemia bargains the cell oxygen transparency and causes hurt that goes from harm to essential organs to a possibly unsafe condition, in general, iron lacking patients should be fortified dependent on their $\mathrm{Hb}$ level, which can uctuate step by step. Iron inadequacy is surveyed by evaluating the degree of $\mathrm{Hb}$, a protein in the red platelet that is the most dependable pointer of Anemia, since Hb supplies oxygen to the amount of the cells of the body. Standard clinical strategies to dismember Anemia basically depend upon the interfering affirmation of blood $\mathrm{Hb}$, which requires venipuncture performed by a clinical administrator or a subject matter expert. Visit blood evaluating prompts the patient's hopelessness, and the need of setting out to an assessment spot or calling a clinical watchman fuses basic cost. Without a doubt, it is of brain blowing fervor to consider procedures and to configuration instruments to screen the mixing of $\mathrm{Hb}$ in a noninvasive manner, with diminished costs, both in the lab and at the patient's home, by and large even bit by bit. Different evaluations show extraordinary energy for the whiteness of body parts to study Anemia. For instance, Aggarwal et al. [22] dissected the credibility of palmar whiteness for the finding of Anemia among kids created 659 months. Tsumura et al. [23] considered skin covering and surface appraisal/combination by dispensing with $\mathrm{Hb}$ and melanin data in the skin. Likewise, [24] reports centers around the relationship among skin and Hb. Spinelli et al. [25] looked at the appraisal of palmar and conjunctival whiteness to recognize Anemia: they found that there was a more obvious degree of affectability to conjunctival paleness than to palmar whiteness. Different assessments discovered associations between clinical signs, for example, paleness to Anemia; in [26], Kalter et al offer the convenience of clinical hints in the revelation of Anemia, which proposes the opportunity of adding conjunctival whiteness to the Integrated Management of Childhood Illness (IMCI), a structure expected to decrease kid mortality and somberness in making nations. Hasan et al [27] and Wang [28], [29] present picture preparing of a ngertip video to investigate the relationship between the picture pixel data and specific $(\mathrm{Hb})$ levels. Furthermore, for the present situation, the proportion of $\mathrm{Hb}$ is ardently inuenced by the shade of the skin or by fundamental tanning. For a long time, experts in like way practice have surveyed Anemia in individuals by review the eyelid conjunctiva. This planning is so far interminable in various miracle locale of the earth. Review the eyelid conjunctiva can offer data to associating Anemia, as two or three assessments proposed. In [30], the nearness of conjunctival whiteness was used for $\mathrm{Hb}$ certification, and the creators appeared at $95 \%$ detachment exactness among iron lacking and non-slight patients subject to an assessment of conjunctival paleness, considering a cut-off of $90 \mathrm{~g} / \mathrm{L}$ for the $\mathrm{Hb}$ fixation. A comparative system is portrayed in [31], The closeness of iron insufficiency in an individual can be an indication of various disorders like jaundice and nonappearance of sustenance. The area of iron insufficiency can serve to show the proximity of various sicknesses moreover. The remainder of the paper is coordinated as follows. Essential writing review related past exploration on Medical Tool to Estimate Anemia are given in II recognition based past work are given in segment ii while segment III Describes Methodology and Implementation For The Previous Existing Approaches. Exploratory outcomes and its examination are given in segment IV. At long last, area V closes the paper.

\section{LITERATURE REVIEW}

A New Method and a Non-Invasive Device to Estimate Anemia Based on Digital Images of the Conjunctiva Anemia is a general clinical issue with basic ramifications for human flourishing. The World Health Organization (WHO) surveys that around two billion individuals are iron deficient, which is dened as Hb focuses that are under the proposed edges [1], [2]. Delicacy is dened as a lessening in the red platelets drifting in the blood or as an abatement in the centralization of Hb: in this manner, it is a reduced restriction of the blood to move oxygen. It begins basically from sound variables (iron deciency, enhancements and minerals), overpowering illnesses (wild fever, intestinal parasites) or acquired fragments (hemoglobin opathies) [3], [4]. These variables can happen in detachment yet are continually related. Iron deciency Anemia is the most eminent dietary deciency, and it causes incalculable passings (allianceanemia.org); it is moreover in danger for broadened horror and mortality in pre-more energetic understudies and pregnant ladies. In 2002, iron deciency Anemia was viewed as among the fundamental contributing parts to the general heap of illness [5]. Inadequacy has a moderate progress: conventionally, no conspicuous signs show up for $\mathrm{Hb}>9-10 \mathrm{~g} / \mathrm{dl}$, since the human body executes compensatory portions, for example, developing the extent of blood siphoned, so the extent of oxygen delivered to the tissues remains essentially unaltered. Precisely when the pay can by and by don't ensure a decent piece of oxygen, signs, for example, inadequacy, whiteness, prickliness, broadened heartbeat, absence of rest, cerebral torture and different others show up. The clinical report, as a rule, is vague and difcult to depict. Moreover, Anemia is reliably not separated or enough treated in never-ending patients for whom it is a tremendous peril factor (counting mortality) and impacts the individual satisfaction. Regardless of its etiology, since an unprecedented level of Anemia bargains the cell oxygen accessibility and causes hurt that goes from naughtiness to basic organs to a possibly dangerous condition, taking everything into account, iron deficient patients should be reinforced dependent on their $\mathrm{Hb}$ level, which can uctuate one small step at a time. Iron need is surveyed by evaluating the degree of $\mathrm{Hb}$, a protein in the red platelet that is the most dependable pointer of Anemia, since Hb supplies oxygen to the entire of the cells of the body. Standard clinical strategies to examine Anemia basically depend upon the intrusive affirmation of blood $\mathrm{Hb}$, which requires venepuncture performed by a clinical manager or a trained professional. Visit blood evaluating prompts the patient's torment, and the need of leaving to an investigation network or calling a clinical watchman consolidates 
basic cost. Without a doubt, it is of mind-blowing energy to consider methodologies and to configuration instruments to screen the mixing of $\mathrm{Hb}$ in a noninvasive manner, with decreased costs, both in the lab and at the patient's home, generally even bit by bit. Different evaluations show incredible energy for the whiteness of body parts to review Anemia. For instance, Aggarwal et al. [6] investigated the realness of palmar whiteness for the finding of Anemia among kids created 659 months. Tsumura et al. [7] considered skin disguising and surface appraisal/blend by wiping out $\mathrm{Hb}$ and melanin data in the skin. What's more, [8] reports centers around the relationship among skin and Hb. Spinelli et al. [9] looked at the evaluation of palmar and conjunctival whiteness to perceive Anemia: they found that there was a more obvious degree of affectability to conjunctival paleness than to palmar whiteness. Different assessments discovered associations between clinical signs, for example, paleness to Anemia; in [10], Kalter et al offer the help of clinical hints in the disclosure of Anemia, which proposes the opportunity of adding conjunctival whiteness to the Integrated Management of Childhood Illness (IMCI), a system expected to diminish kid mortality and somberness in making nations. Hasan et al [11] and Wang [12], [13] present picture arranging of a ngertip video to investigate the relationship between the picture pixel data and indisputable (Hb) levels. Moreover, for the present condition, the check of $\mathrm{Hb}$ is unfalteringly inuenced by the shade of the skin or by principal tanning. For a long time, experts in like way practice have evaluated Anemia in individuals by review the eyelid conjunctiva. This arrangement is so far immense in various surprise regions of the earth. Survey the eyelid conjunctiva can offer data to associating Anemia, as two or three assessments recommended. In [14], the vicinity of conjunctival whiteness was used for $\mathrm{Hb}$ assertion, and the creators appeared at 95\% confinement accuracy among iron lacking and non-fragile patients dependent on an assessment of conjunctival paleness, considering a cut-off of $90 \mathrm{~g} / \mathrm{L}$ for the $\mathrm{Hb}$ fixation. A comparable way of thinking is portrayed in [15]

\section{REASEARCH GAP}

As we probably areaware, we are living in the period of 3D clinical science where we have bunches of Tools which can distinguish the infection. In present period we have bunches of innovation yet there is absence of clinical devices which can discover paleness with any intrusive cycle. At present we need to take the blood tests and through that we can discover the paleness. At present there is heaps of exploration who are dealing with Non obtrusive cycle where they are utilizing the idea of picture preparing. Here they are utilizing the methodologies like eye side or nails. So according to the momentum search there is loads of examination holes are there which is truly should be fathomed and those are followings:

- $\quad$ Lack of Real Time: All current methodology are require pre characterize pictures of eye side or nails, so for that approach we need to catch great quality picture through eye side scanner or top of the line camera.

- Accuracy: All current methodologies can't make appropriate outcome as they are center around one single methodology like SPO2 based, EYE side dependent on nails based so there is any cross-check technique is accessible which give guarantee of weakness.

- Long Process: according to scanner approach all past existing devices are require aches time

- $\quad$ Time Issue: All current calculation rush to give appropriate outcome as expected.

- Quality Issue: This is a major peril which is truly should be settled there is no any methodology which can get qulity level report from the information boundaries.

\section{Methadology}

In this segment we talk about the fundamental Anemia assessment measure, what sort of essential calculation was utilized and what are the development research is there. Here we did the total near investigation and usage of those methodologies. These are the previous approach which are working in this area and we did the comparative analysis with these approaches.
a. Eye Side Based [30]
b. Nails Based [35]
c. Spo2 based [36]
d. Color Analysis [39]

4.1 Giovanni [30]: He author propose a non-invasive way to deal with $\mathrm{Hb}$ estimation. 


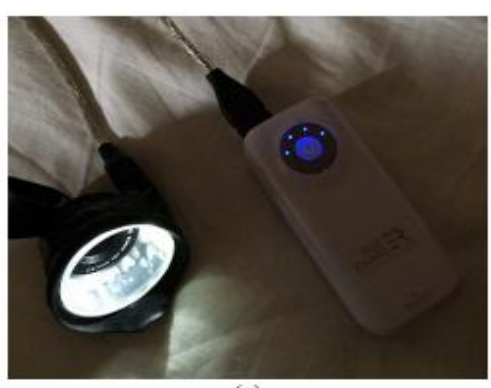

(a)

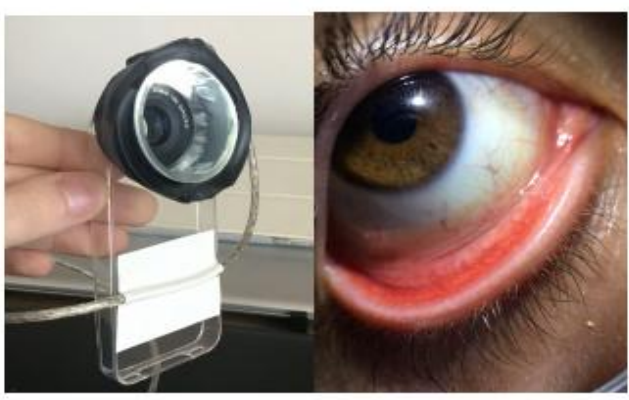

(b)

(c)

Fig. 4.1 Eye Based Anemia Detection[30]

4.2Trupti [35]:In this work creator manages in such situation to take choice in malady finding. The contribution to the proposed framework is individual nail picture.

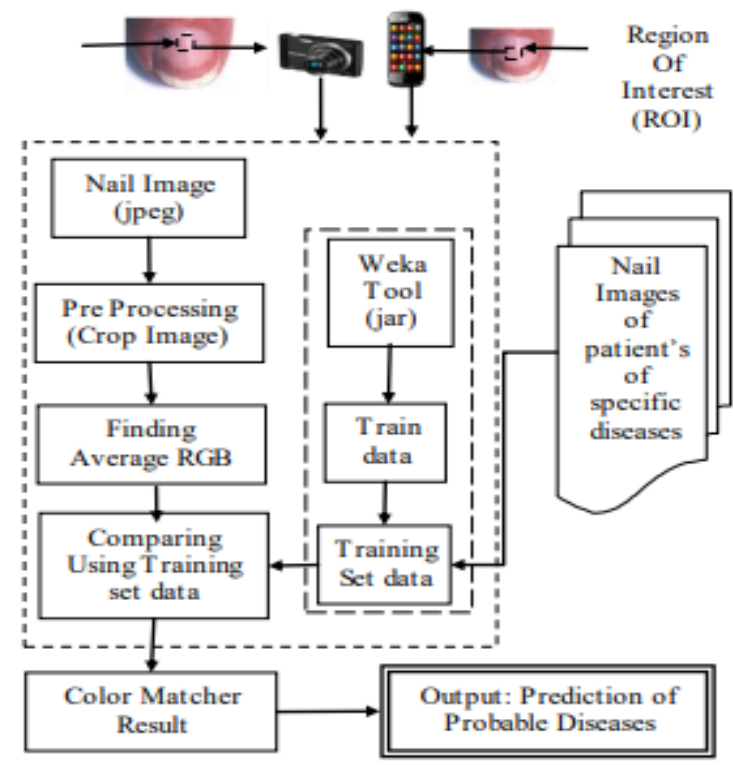

Fig. 4.2 Nail Based Approach [35]

4.3Komal [36]: In this work author create a system which is able to detect the blood oxidation level Using SPO2. 


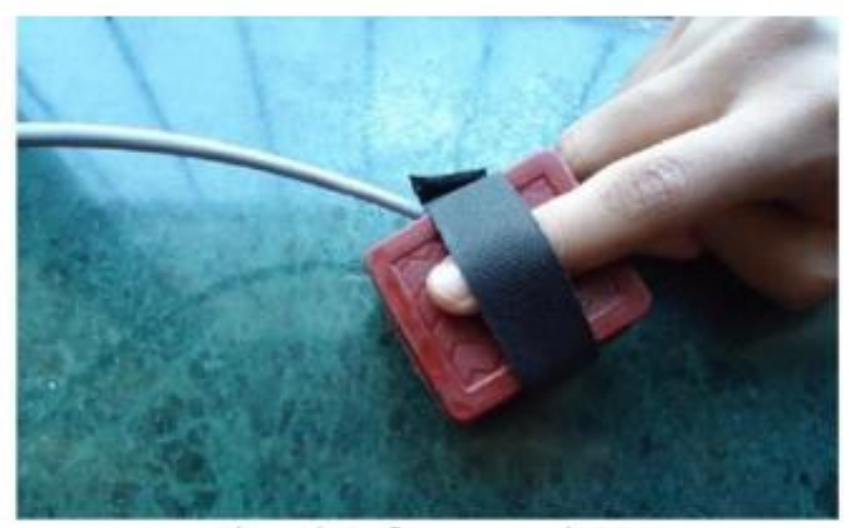

Fig. 4.3 SPO2 Based Approach [36]

4.4Srinivasan[39]: In this work author create a system which will take the photograph of the thumb with blood\& based on color classification analysis the anemia.

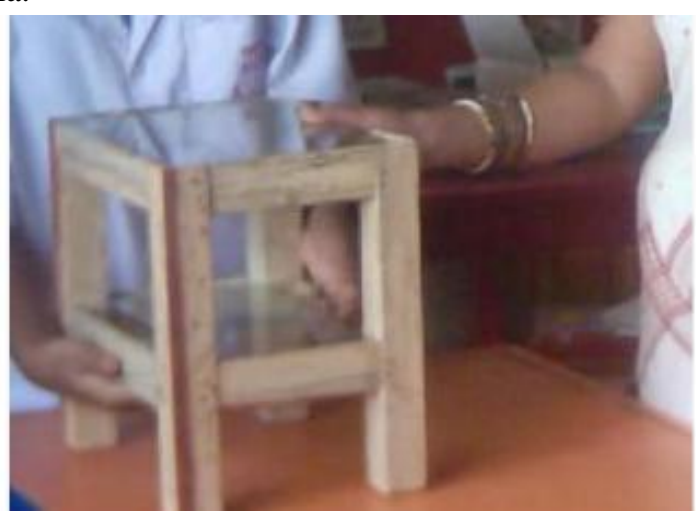

Fig. 4.4 Blood Based approach [39]

\section{Proposed Algorithm:}

As we know present era there is need of fast processing system so this is our proposed approach for the anemia detection: In this approach we use the Raspberry Pi Zero with 5M pixel 1080P camera, using that we take the real time image of the user nail and based on that image we apply the color classification and image classification approach to detect the anemia level, here we use the deep learning \& machine learning approach so for that we train our system with existing image data base and based on that our system is able to give the more accurate result.

\section{Proposed Algorithm:}

\{

Start:

Real Time Image Capture Using PI Cam

Image Analysis

RGB color Analysis

Separation of R G \& B

Pre-Analysis of Image

Creating Data base for R G \& B

Creating Partanen on R G \& B

Image Classification Using Deep Learning

define a set of target classes

Creating a train model to recognize nail

Color Classification Using Deep Learning 


\section{define a set of target classes}

Creating a train model to recognize nail color

Training Process for the color \& nail Data Set Using Pillow

\section{Comparison with Train Data Set Using Pillow}

\section{Color Analysis}

Result

\section{Stop}

\}

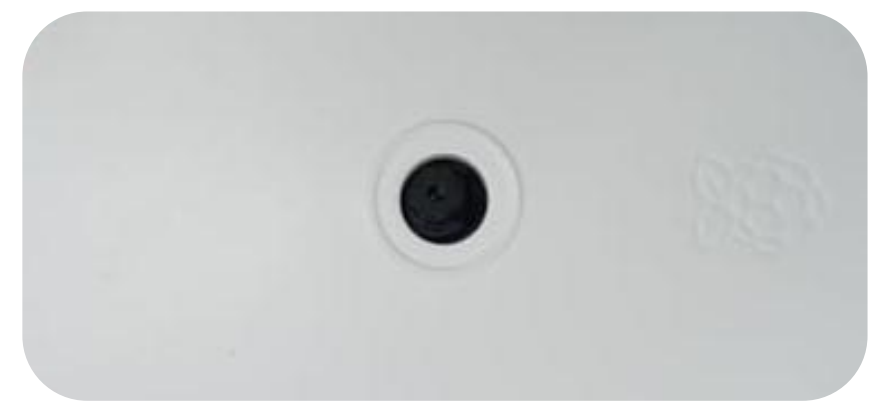

Fig. 4.5 Device Design

\section{RESUlT \& ANALYSIS}

In this section we introduce the relative investigation of all with past existing methodology. As we can see table. 5.1 shows the comparative analysis based on the some of the parameters which are followings:

1. Efficiency

2. Cost Complexity

3. Error Level (\% Accuracy)

4. Time

Table 4.1 Comparative Analysis

\begin{tabular}{|c|c|c|c|c|}
\hline Process & Cost & Efficiency & \% Accuracy & Time \\
& & & & \\
\hline Giovanni & Below Average & High & High & Less \\
\hline Trupti & Low & Average & High & Very Less \\
\hline Komal & High & Very High & Average & High \\
\hline Srinivasan & Average & Mid & Low & Normal \\
\hline Proposed & Average & Average & Very High & Low \\
\hline
\end{tabular}


Here table 4.1 shows the shows the near investigation of time, Efficiency, exactness and gadget necessity dependent on the similar we can say Proposed approach is hitter regarding all boundaries as it requires less time and high precision is normal cost the expense of framework, according to relative examination our proposed approach is obviously better than the current methodology.

\section{CONCLUSION}

2020 is referred to for COVID-19 as we probably are aware because of COVID-19 heaps of clinical issues are made. Paleness is exceptionally unsafe for COVID-19 the individual who have frailty they have high opportunity to get the influenced by the COVID-19, so as we probably are aware to identify Anemia it's a troublesome cycle, client needs to go to the lab and afterward just they will get the outcome which is risky in this work we proposed a quick handling calculation which is work on continuous our proposed calculation can recognize the sickliness. Here we additionally did the near investigation and dependent on the examination we discovered our proposed approach is superior to the all-past existing methodology.

\section{REFERENCES}

[1] Assessing the Iron Status of Populations, World Health Org., Geneva, Switzerland,2004.

[2] World Health Organization, Worldwide Prevalence of Anaemia 1993- 2005: WHO Global Database on Anaemia, B. de Benoist, E. McLean, I. Egli, and M. Cogswell, Eds. Geneva, Switzerland: World Health Organization,2008.

[3] E. McLean, M. Cogswell, I. Egli, D.Wojdyla, and B. de Benoist, "Worldwide prevalence of anaemia, WHO vitamin and mineral nutrition information system, 1993-2005," Public Health Nutrition, vol. 12, no. 4, pp. 444454, 2009.

[4] World Health Organization, “Iron deciencyanaemia: Assessment, prevention and controlA guide for programme managers," WHO Reference WHO/NHD/01.3, 2001. [Online]. Available: http://www.who.int/nutrition/topics/ida/en/

[5] The World Health Report 2002: Reducing Risks, Promoting Healthy Life, World Health Org., Geneva, Switzerland,2002.

[6] A. K. Aggarwal, "Validity of palmar pallor for diagnosis of anemia among children aged659 months in North India," Anemia, vol. 2014, Nov. 2014, 2 Art. $543860 . \quad$ [Online].

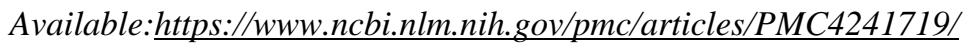

[7] N. Tsumura et al., "Image-based skin color and texture analysis/synthesis by extracting hemoglobin and melanin information in the skin," ACM Trans. Graph., vol. 22, no. 3, pp. 770779, 2003.

[8] E. Angelopoulou, "Understanding the color of human skin," Proc. SPIE, vol. 4299, pp. 243251, Jun. 2001.

[9] M. G. N. Spinelli, J. M. P. Souza, S. B. de Souza, and E. H. Sesoko, “Reliability and validity of palmar and conjunctival pallor for anemia detection purposes," Rev. Saude Publica, vol. 37, no. 4, pp. 404408, 2003.

[10] H. Kalter et al., "Evaluation of clinical signs to diagnose anaemia in uganda and Bangladesh, in areas with and without malaria," Bull. World Health Org., vol. 75, no. 1, p. 103, 1997.

[11] M. K. Hasan, N. Sakib, R. R. Love, and S. I. Ahamed, “RGB pixel analysis of ngertip video image captured from sickle cell patient with low and high level of hemoglobin," in Proc. IEEE 8th Annu. Ubiquitous Comput., Electron. Mobile Commun. Conf. (UEMCON), Oct. 2017, pp. 499505, doi:10.1109/UEMCON.2017.8249004.

[12] E. J. Wang, W. Li, D. Hawkins, T. Gernsheimer, C. Norby-Slycord, and S. N.Patel,

"HemaApp: Noninvasive blood screening of hemoglobin using smartphone cameras," in Proc.ACM Int. Joint Conf. Pervasive Ubiquitous Comput. (UbiComp), 2016, pp. 593604, doi: 10.1145/2971648.2971653.

[13] E. J. Wang, W. Li, J. Zhu, R. Rana, and S. N. Patel, “Noninvasive hemoglobin measurement using unmodied smartphone camera and white ash," in Proc. 39th Annu. Int. Conf. IEEE Eng. Med. Biol. Soc. (EMBC), Seogwipo, South Korea, Jul. 2017, pp. 23332336, doi: 10.1109/EMBC.2017.8037323.

[14] T. N. Sheth, N. K. Choudhry, M. Bowes, and A. S. Detsky, 'The relation of conjunctival pallor to the presence of 
anemia," J. Gen. Internal Med., vol. 12, no. 2, pp. 102106,1997.

[15] R. M. D. Silva and C. A. Machado, "Clinical evaluation of the paleness: Agreement between observers and comparison with hemoglobin levels," Rev. Brasileira Hematol. Hemoterapia, vol. 32, no. 6, pp. 444448,2010.

[16] C. I. Sanchez-Carrillo, T. de Jesus Ramirez-Sanchez, and B. J. Selwyn, "Test of a noninvasive instrument for measuring hemoglobin concentration," Int. J. Technol. Assessment Health Care, vol. 5, no. 4, pp. 659667,1989.

[17] S. Suner, G. Crawford, J. McMurdy, and G. Jay, "Non-invasive determination of hemoglobin by digital photography of palpebral conjunctiva," J. Emerg. Med., vol. 33, no. 2, pp. 105111, 2007.

[18] Y.-M. Chen, S.-G. Miaou, and H. Bian, “Examining palpebral conjunctiva for anemia assessment with image processing methods," Comput. Meth- ods Programs Biomed., vol. 137, pp. 125135, Dec. 2016, doi: 10.1016/j.cmpb.2016.08.025.

[19] Y.-M. Chen and S.-G. Miaou, “A Kalman ltering and nonlinear penalty regressionapproach for noninvasive anemia detection with palpebral conjunctiva images," J. Healthcare Eng., vol. 2017, Jul. 2017, Art. no. 9580385. [Online]. Available: https://www.hindawi.com/ journals/jhe/2017/9580385/cta/, doi:10.1155/2017/9580385.

[20] O. Kim, J. McMurdy, G. Jay, C. Lines, G. Crawford, and M. Alber, "Combined reectance spectroscopy and stochastic modeling approach for noninvasive hemoglobin determination via palpebral conjunctiva," Physiol. Rep., vol. 2, no. 1, p. e00192,2014.

[21] V. Bevilacqua et al., "A novel approach to evaluate blood parameters using computer vision techniques," in Proc. IEEE Int. Symp. Med. Meas. Appl. (MeMeA), Benevento, Italy, May 2016, pp. 16, doi:10.1109/MeMeA.2016.7533760.

[22] H. S. Wu, J. Barba, and J. Gil, "Iterative thresholding for segmentation of cells from noisy images" Journal of Microscopy, vol. 197, no. 3, pp. 296-304,2000

[23] C. Di Rubeto, A. Dempster, S. Khan, and B. Jarra, "Segmentation of blood images using morphological operators," in Proceedings of the 15th International Conference on Pattern Recognition, vol. 3, pp. 397-400, 2000.

[24] C.D., Ruberto, A., Dempster, S., Khan, B.Jarra, "Analysis of Infected Blood Cell Images Using Morphological Operators”, Image and Vision Computing, Vol. 20, 2002,pp. 133-146

[25] Q. Liao and Y. Deng, “An accurate segmentation method for white blood cell images, " in Proceedings of the IEEE International Symposium on Biomedical Imaging, pp. 245-258, 2002

[26] J.L. Starck, E.J. Candes, D.L. Donoho, “The curvelet transform for image denoising”, IEEE Transaction on Image Processing 11 (6) (2002)670-684.

[27] G. Lin, U. Adiga, K. Olson, J. F. Guzowski, C. A. Barnes, and B. Roysam, "A hybrid 3Dwatershed algorithm incorporating gradient cues and object models for automatic segmentation of nuclei in confocal image stacks, ” Cytometry Part A, vol. 56, no. 1, pp. 23-36, 2003.

[28] Fabio Scotti University of Milan, Department of Information Technologies, via Bramante 65, 26013 “Automatic Morphological Analysis for Acute Leukemia Identification in Peripheral Microscope Images" IEEE International Conferenceon Computational Intelligence For Measurement Systems and Giardini Naxos, Italy, 20-22 July2005.

[29] Muthalagu, R., V. Thulasi Bai, and Sheila John. "A smart (phone) solution: An effective tool for screening anaemiacorrelation with conjunctiva pallor and haemoglobin levels." TAGA Journal 14 (2018):2611-2621.

[30] Dimauro, G.; Caivano, D.; Girardi, F. A New Method and a Non-Invasive Device to Estimate Anemia Based on Digital Images of the Conjunctiva. IEEE Access 2018, 6, 46968-46975, doi:10.1109/access.2018.2867110.

[31] Tyagi, Megha. "Detection of Poikilocyte Cells and Haemoglobin Using Digital Image Processing."(2018). 
[32] Anand, Bhavya, Yukta Goel, and Amita Goel. "RED BLOOD CELL DETECTION USING HAUGH TRANSFORM AND SURF TECHNIQUE." RED 7.3(2018).

[33] Tamir, Azwad, et al. "Detection of anemia from image of the anterior conjunctiva of the eye by image processing and thresholding." Humanitarian Technology Conference (R10-HTC), 2017 IEEE Region 10. IEEE, 2017.

[34] Bhanushali, Akshay, et al. "Automated Disease Diagnosis Using Image Microscopy." (2016).

[35] Indi, Trupti S., and Yogesh A. Gunge. "Early stage disease diagnosis system using human nail image processing." IJ Information Technology and Computer Science 7 (2016):30-35.

[36] Bhatia, Komal, and Mandeep Singh. "Non-Invasive Techniques for Detection of Hemoglobin in Blood: A Review."

[37] Hasan, Md Kamrul, et al. "Analyzing the existing noninvasive hemoglobin measurement techniques." Ubiquitous Computing, Electronics and Mobile Communication Conference (UEMCON), 2017 IEEE 8th Annual. IEEE,2017.

[38] Collings, Shaun, et al. "Non-invasive detection of anaemia using digital photographs of the conjunctiva." PloS one 11.4 (2016):e0153286.

[39] Srinivasan, K. S., et al. "Non-invasive estimation of hemoglobin in blood using color analysis." First International Conference on Industrial and Information Systems. IEEE,2006. 